\section{Unter die Haut}

Subkutan (s. c.) verabreichtes Bortezomib zur Behandlung des multiplen $\mathrm{My}$ eloms (MM) ist genauso wirksam wie intravenös (i.v.) verabreichtes, dabei aber besser verträglich. Das bestätigte die Nachbeobachtung der Phase-IIIStudie MMY-3021 mit Patienten mit MM und mindestens einer Vortherapie [Arnulf B et al. Haematologica. 2012; 97(12):1925-8]. Nach über 17 Monaten und bis zu 10 Zyklen Bortezomib mit und ohne Dexamethason lag das Ansprechen bei $52 \%$ in jedem Arm, wobei die Rate eines kompletten oder fast kompletten Ansprechens bei Bortezomib s.c. $23 \%$ und bei i.v.-Applikation $22 \%$ betrug. Die mediane Zeit bis zur Progression war mit 9,7 bzw. 9,6 Monaten ebenso vergleichbar wie das mediane progressionsfreie Überleben $(9,3$ vs. 8,4 Monate) und das 1-Jahres-Gesamtüberleben $(76,4$ vs. $78,0 \%)$. Im Studienarm mit Subkutangabe von Bortezomib waren aber die Neuropathieraten signifikant geringer und verschwanden auch häufiger und schneller als bei i.v.-Applikation.

red

Nach Information von Janssen-Cilag

\section{Milzgröße im Visier}

Der selektive JAK1- und JAK-2-Hemmer Ruxolitinib (Jakavi ${ }^{\oplus}$ ) verringert nach Daten, die beim Kongress der amerikanischen Hämatologiegesellschaft ASH im Dezember vorgestellt wurden, die Milzgröße bei Myelofibrose mit mittlerem oder hohem Risiko und Splenomegalie anhaltend [Cervantes F et al. Blood. 2021;120(21):Abstr. 801]. Die bei 48,3\% der Patienten erreichte mindestens $35 \%$ ige Reduktion der Milzgröße blieb bei Ruxolitinib-Behandlung nach einer Nachbeobachtungszeit von 48 und 84 Wochen mit einer hohen Wahrscheinlichkeit erhalten (75 und $58 \%$ ). Das scheint sich im Trend auch in einen Überlebensvorteil $\mathrm{zu}$ übersetzen, wie die 2-Jahres-Daten zeigten (Hazard Ratio 0,52 für Ruxolitinib gegenüber bester verfügbarer Therapie; $95 \%$-Konfidenzintervall 0,27-1,00).

Metastasiertes kolorektales Karzinom

\title{
Neuer Ansatz für die Second-Line-Therapie
}

Der Anti-VEGF-Antikörper Bevacizumab (Avastin ${ }^{\oplus}$ ) kann das Gesamtüberleben der Patienten nicht nur in der Erstlinie bis zur Progression verlängern, sondern wirkt auch über mehrere Therapielinien.

Dass die Wirksamkeit von Bevacizumab über mehrere Therapielinien erhalten bleibt, belegen die Ergebnisse der PhaseIII-Studie TML (Treatment through Multiple Lines)-Studie, an der 820 Patienten mit metastasiertem Kolorektalkarzinom (mCRC) teilnahmen [Bennouna J et al. Lancet Oncol. 2013;14(1):29-37]. Die Erstlinientherapie hatte aus Bevacizumab kombiniert mit einer Oxaliplatinoder Irinotecan-basierten Chemotherapie bestanden. Nach Krankheitsprogression erhielten die Patienten randomisiert erneut eine Bevacizumab-kombinierte Chemotherapie oder eine alleinige Chemotherapie, wobei die in der Erstlinie mit Oxaliplatin behandelten Patienten in der Zweitlinie ein Irinotecan-basiertes Regime erhielten und umgekehrt. Die in der Zweitlinie fortgeführte Gabe des Angiogenesehemmers war mit einer signifi- kanten Verbesserung des Gesamtüberlebens assoziiert. Bei Bevacizumab-Kombinationstherapie betrug das mediane Gesamtüberleben 11,2 Monate, bei alleiniger Chemotherapie 9,8 Monate (Hazard Ratio [HR] 0,81; $\mathrm{p}=0,0062$ ). Das progressionsfreie Überleben betrug 4,1 Monate unter alleiniger Chemotherapie und 5,7 Monate mit Bevacizumab (HR 0,68; $\mathrm{p}<0,0001)$. Die Tumorkontrollrate lag in der Bevacizumab-Gruppe mit $68 \%$ über der in der Vergleichsgruppe mit $54 \%(\mathrm{p}<0,0001)$. Wie Stefan Kubicka, Reutlingen, herausstellte, wurde Bevacizumab auch bei fortgesetzter Gabe gut vertragen.

Abdol A. Ameri

Pressekonferenz "Avastin ${ }^{\circledast}$ beim metastasierten Kolorektalkarzinom (mCRC) - Therapie über die erste Tumorprogression hinaus". Berlin,

17.01.2013; Veranstalter Roche Pharma

\section{Neue Chance vor der Chemo}

\section{War Abirateronacetat (Zytiga ${ }^{\oplus}$ ) beim metastasierten, hormonresistenten Prostatakarzinom (mHRPC) bisher nur bei Versagen der Docetaxel-haltigen Chemotherapie zugelassen, wurde die Indikation jetzt auf chemotherapie- naive Patienten erweitert.}

Basis der Zulassungserweiterung ist die Phase-III-Studie COU-AA-302, an der 1.088 chemotherapienaive asymptomatische oder mild symptomatische Patienten mit mHRPC teilnahmen [Ryan CJ et al. N Engl J Med. 2013;368(2):138-48]. Sie erhielten randomisiert entweder Abirateronacetat plus Prednison/Prednisolon oder Placebo und Predison/Prednisolon.

Nach einer geplanten Zwischenauswertung zeigte sich ein deutlicher Vorteil der Abirateronacetat-Therapie mit einem medianen radiologischen progressionsfreien Überleben von 16,5 vs. 8,3 Mona- ten (Hazard Ratio [HR] 0,53; p < 0,001). Nach einer medianen Nachbeobachtungszeit von 22,2 Monaten war zudem das Gesamtüberleben signifikant besser: Das Median unter Abirateron war noch nicht erreicht, in der Kontrollgruppe lag es bei 27,2 Monaten (HR 0,75; p = 0,01). Die Kombination von AbirateronacetatPrednison verzögerte außerdem den Beginn der zytotoxischen Chemotherapie, der Schmerztherapie mit Opiaten, des PSA-Progresses und der Reduktion des Allgemeinzustands. Dabei waren steroidabhängige Nebenwirkungen des Grads 3 oder 4 inklusive kardiale Erkrankungen (6 vs. $3 \%$ ) sowie abnorme Leberwerte (ALT-Erhöhung 5 vs. $3 \%$; AST-Erhöhungen 3 vs. < $1 \%$ ) in der Abirateron-Gruppe etwas häufiger als bei den Kontrollen.

red

Nach Informationen von Janssen-Cilag 